\title{
Passive BCI in Operational Environments: Insights, Recent Advances and Future trends
}

\author{
Pietro Aricò ${ }^{*}$, Gianluca Borghini ${ }^{\S}$, Gianluca Di Flumeri ${ }^{\S}$, Member, IEEE, Nicolina Sciaraffa, \\ Member, IEEE, Alfredo Colosimo and Fabio Babiloni, Member, IEEE
}

\begin{abstract}
Goal: this mini-review aims to highlight recent important aspects to consider and evaluate when passive BrainComputer Interface (pBCI) systems would be developed and used in operational environments, and remarks future directions of their applications. Methods: Electroencephalography (EEG)-based pBCI has become an important tool for real-time analysis of brain activity, since it could potentially provide, covertly - without distracting the user from the main task - and objectively - not affected by the subjective judgement of an observer or the user itself - information about the operator cognitive state. Results: different examples of pBCI applications in operational environments and new adaptive interface solutions have been presented and described. In addition, a general overview regarding the correct use of machine learning techniques (e.g. which algorithm to use, common pitfalls to avoid, etc.) in the pBCI field has been provided. Conclusion: despite recent innovations on algorithms and neuro-technology, pBCI systems are not completely ready to enter the market yet, mainly due to limitations of the EEG electrodes technology, and algorithms reliability and capability in real settings. Significance: high complexity and safety critical systems (e.g. airplanes, ATM interfaces) should adapt their behaviors and functionality accordingly to the user' actual mental state. Thus, technologies (i.e. pBCIs) able to measure in real-time the user's mental states would result very useful in such "high risk" environments to enhance human machine interaction, and so increase the overall safety.
\end{abstract}

Index Terms-Human-Machine Interaction (HMI), Adaptive Automation, Machine learning techniques, Mental states, operational environments, passive Brain-Computer Interface (pBCI).

\section{INTRODUCTION}

I $\mathrm{N}$ several complex and safety-critical domains, such as aviation, health-care systems and self-driving cars, the role of humans has shifted from total manual control to passive monitoring of autonomous systems operating together, such as autopilots (in aircraft, cars, and other means of transport), automated collision avoidance systems (in air-traffic control and driving), automated production cycles (in industry and

Date of submission: 2016-12-22

"This work is co-financed by the European Commission by Horizon2020 projects Sesar-01-2015 Automation in ATM, "Stress", GA n. 699381 and Sesar-06-2015 High Performing Airport Operations, "MOTO”, GA n. 699379. The grant provided by the Italian Minister of University and Education under the PRIN 2012, GA n. 2012WAANZJ scheme to F.B. is also gratefully acknowledged."

*P. Aricò is with the Dept. Molecular Medicine, Sapienza University of Rome, Italy and BrainSigns Co. Ltd, Rome, Italy (correspondence e-mail: energy plants), and so on. The increasing complexity of automation makes human monitoring and intervention a difficult task, especially when humans are overwhelmed with multiple sources of information on the same perceptual channels, e.g., two visual stimuli. In addition, new technology, when introduced poorly (i.e. inadequate training of the operator), could induce overload conditions in the operator, and consequently increase the risk of errors commission [1]. In the recent years, it has been explored the possibility to quantify in real-time the actual cognitive state (e.g. workload, attention) of the operator during his/her working activity by using passive Brain-Computer Interface (pBCI) technologies. Such information could be then used to change/adapt the behavior of the interface that the user is interacting with, in order to avoid, or at least mitigate error commission risk, and more in general to improve Human Machine Interaction (HMI).

In this mini-review, we would point out some recent advancements regarding pBCI technologies and recommendations for their employment in operational environments. Firstly we introduced electrophysiological measures for the human psychophysiological state evaluation. Then, we focused on machine learning techniques to be employed in pBCIs and common pitfalls in using such techniques in operational environments. In conclusion, future directions and open issues to make pBCI systems usable in real contexts have been highlighted.

\section{Mental STATES EVALUATION BY USING NEUROMETRICS}

Many electrophysiological measures have been used for the human psychophysiological state evaluation, focusing on brain activity analysis, by means of Electroencephalography (EEG), functional Near-InfraRed spectroscopy (fNIRs), functional Magnetic Resonance Imaging (fMRI), Magnetoencephalography (MEG), and other types of biosignals, such as Electrocardiography (ECG), Electrooculography (EOG) and Galvanic Skin Response (GSR) (e.g. [2]). In the perspective of applying electrophysiological

pietro.arico@uniroma1.it). G. Borghini is with Dept. Molecular Medicine, Sapienza University of Rome, Italy BrainSigns Co. Ltd, Rome, Italy, and IRCCS Fondazione Santa Lucia, Rome, Italy. G. Di Flumeri, N. Sciaraffa and A. Colosimo are with Dept. Anatomical, Histological, Forensic \& Orthopedic Sciences, Sapienza University of Rome, Italy. F. Babiloni is with the Dept. Molecular Medicine, Sapienza University of Rome, Italy and BrainSigns Co. Ltd, Rome, Italy.

$\S$ These authors contributed equally to this work. 
measures during everyday-life activities, issues related to the size, weight, and power of equipment do not allow the use of MEG and fMRI [3]. In this regard, different electrophysiological indicators have been tested in ecological settings, within the aviation domain (both military and civil by using EEG, fNIRs, ECG, EOG and pupil dilation), surgery (EEG), sport (EEG), city traffic monitoring (EEG, fNIRS, ECG, Eye tracking), plants control centers (EEG, ECG) and other day-life activities (EEG, ECG, EOG, fNIRS, GSR, e.g. [4]). Summarizing the evidences, EOG, ECG, GSR and ocular activities measurements highlighted a correlation with some mental states (stress, mental fatigue, drowsiness), but they were demonstrated to be useful and robust only in combination with other neuroimaging techniques directly linked to the Central Nervous System (CNS), i.e. the brain [2]. Consequently, the EEG and fNIRs are the most likely candidates that can be straightforwardly employed to realize HMI applications usable in operational environments, although fNIRs seems to be characterized by a lower sensitivity toward brain activity variations with respect to EEG techniques [3].

\section{PASSIVE BRAIN-COMPUTER INTERFACE TECHNOLOGIES}

Enhancing user experience is a constant goal for HMI research, and, as stated before, ways to reach this objective are several, from changing the interface properties, to adapt the task to the actual user's ability level [5], which would define an implicit input modality, carrying information about the user state not sent intentionally by her/himself. By measuring different user's covert mental states, such as attention, workload, frustration and stress, the user interface could be immediately adapted to keep operators optimally working. In this regard, in the past few years, Brain-Computer Interface (BCI) technology has gained popularity in the form of measurement devices, allowing to assess and track mental states in real-time, and to use them with the aim i) to provide a feedback to the user, and/or ii) to modify in a closed loop the behavior of the interface that the user is interacting with [4]. Such kind of BCI has been called passive BCI (pBCI). With respect to the classical definition of $\mathrm{BCI}$, that aims at providing a communication channel for users based on the spontaneous (Sensory motor rhythms based BCIs) or induced (i.e. P300 or SSVEP based BCIs) modulation of the brain signals, so called pBCIs [6] make use of spontaneously generated brain signals modulated by the actual mental status, in order to allow for an online detection of the user's mental state. In addition, such technology could be able to detect specific brain states even before they reach the user's consciousness and before they trigger user's behavioral actions. In other words, a potentially dangerous situation may be detected before the operator starts to perform an action, representing a critical aspect in several safety-critical applications, such as aviation, driving, industrial environments or security surveillance. With respect to driving assistance applications, different studies have investigated the use of pBCIs during driving simulations for assessing driving performance and inattentiveness, and for detecting needs of emergency brakes before the braking onset. For example, Haufe and colleagues [7] implemented and evaluated an online emergency braking detector in a simulation environment, and showed that electrophysiological signals coming from driver's brain and muscles reached the predictive accuracy of behavioral channels at earlier stages of the emergency situation. Authors concluded that the time that can be saved by the system was around $130(\mathrm{~ms})$. At the speed of $100(\mathrm{~km} / \mathrm{h})$, this result means a reduction of the braking distance of $3.66(\mathrm{~m})$. Their preliminary results performed in laboratory settings were then reproduced during real-driving contexts, demonstrating how well-designed simulator studies can be a useful proxy for real world studies. Also, pBCI systems can potentially be used for cognitive real-time monitoring of driver's mental workload, and employing this information to alert the driver and/or to trigger the car behavior when the current level of workload reaches critical levels. In a different context, initial steps have been taken towards assistive technologies that use the current mental state of a user for avoiding accidents in industrial environments. For example, pBCI-related studies have been proposed in aviation field, in particular for air traffic controllers and aircraft pilots, in which mental states of the operators have been evaluated in real-time by using their brain activity, with the purpose i) to quantify the task demand along the workshift [4], ii) to evaluate the training level of the user [8], and iii) to adapt interfaces depending on the actual mental state of the operator (e.g. [5]).

\section{ELECTROPHYSIOLOGICAL FEATURES IDENTIFICATION FOR MENTAL STATES ESTIMATION}

There are several studies that highlighted clear correlations between user mental states and several EEG features variation, both in time and in frequency domain. For example, the theta $(\theta$ : over the frontal) and alpha ( $\alpha$ : over the parietal sites) rhythms of the EEG signal have been taken into account in several studies because of their strong correlation with mental workload variations [2], [9]. Regarding the attention

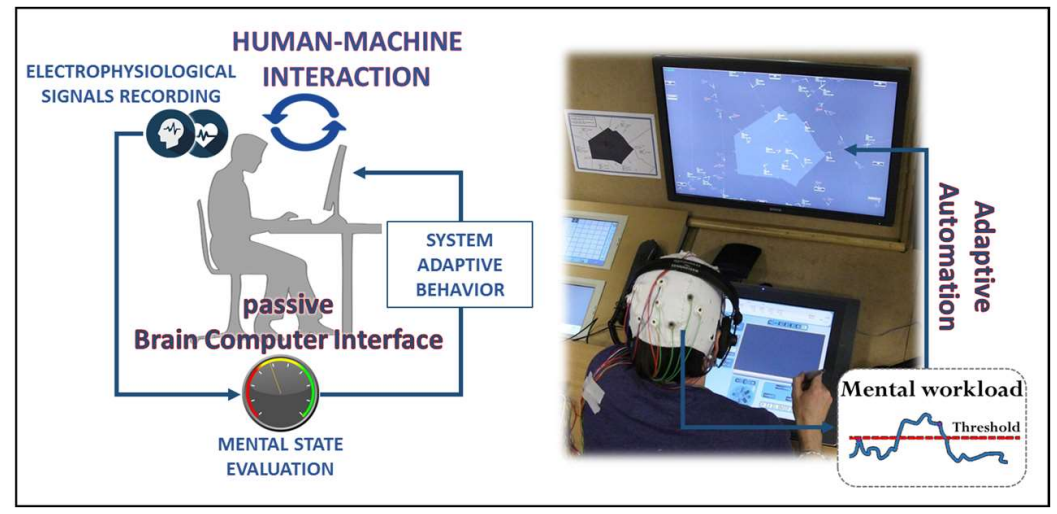

Fig. 1. (Left side) Representation of how passive BCI concept is used to enhance human machine interaction. In particular, the pBCI is able to quantify the actual mental state (e.g. attention, workload) of the user, even in realtime, without interfere with his/her work. Such information can then be used to change the behavior of the user interface accordingly (e.g. adaptive automation in Air Traffic Environment, right side, [5]). 
assessment, the EEG-based event related potential P300 could be considered as a measure of the attentional resources of the subject. In particular, the amplitude of the P300 is proportional to the amount of attentional resources engaged in processing a given stimulus. Further, P300 latency is thought to reflect stimulus classification speed, such that it serves as a temporal measure of neural activity underlying attention allocation and immediate memory operations. Therefore, EEG $\alpha$ power decreases when individuals become engaged in the task and increase their attentional level, as compared to the simple retention of information (e.g. [10]). Another interesting feature that may be used to realize a $\mathrm{pBCI}$ is the error-related negativity (ERN), that is related to perceived accuracy, or the extent to which participants are aware of their errors [11]. The ERN allows identification, prediction and even prevention of operators' errors in real-time. Theoretically, a system could be activated by an ERN detector in order to either take control of the situation (for example in those cases where time to act is very limited), or notifying the operator about the error he/she committed by providing an adaptive interface which selectively presents the critical sub-systems or function. In this regard, the space of the features is multidimensional (i.e. several channels and time/frequencies samples), and the signal-to-noise ratio (SNR) is often low, i.e. in P300 based BCIs [12]. Another promising technique to measure user's mental states is related to the analysis of changes within brain networks connectivity, that allows to deeply investigate interactions across cerebral cortex areas during mental states variations [13]. For instance, Sun et al., [14] developed a functional connectivity-based mental fatigue monitoring method, demonstrating its potential application in operational environments. Another interesting approach regarding the mental workload evaluation has been explored by Dimitriadis and colleagues [15], who proposed a novel functional connectivity-based approach. In particular, they used a Tensor Subspace Analysis (TSA) to represent connectivity data, achieving a high correct-recognition-rate of difficulties in an arithmetic task.

These studies are just some examples of possible EEG features that could be used to realize passive BCI applications with the aim to measure the actual user mental status.

Although such features have been demonstrated to be reproducible across subjects, their estimations are often slow (e.g. more than five minutes in order to highlight differences between different mental workload levels by using EEG frequency bands variations [16]) and inaccurate at level of single subject, thus often not suitable for online applications in operational environments.

\section{MENTAL STATES MEASUREMENT BY USING MACHINE LEARNING}

Machine-learning approaches for mental states estimation based on the analysis of physiological data went through a rapid expansion in the last decades since such methodologies are able to provide the means to decode and characterize task relevant brain states (i.e. reducing from multidimensionality to one dimensionality problem) and to distinguish them from noninformative brain signals (i.e. to enhance SNR). With regard to
BCI applications, few novel data driven approaches specific to neuroscience have emerged in the last decades: (a) dimension reduction and projection methods, (b) classification methods, (c) spatio-temporal filtering algorithms, (d) measures for determining synchrony, coherence or causal relations in data and (e) algorithms for assessing and counteracting nonstationarity in data. In this regard, several studies (e.g. [17]) provided a complete review focused on most widely used and high performing machine learning techniques, so a deep dissertation about their mathematical explanation is out of the scope of this review. On the contrary, it would be important to discuss some important aspects, regarding issues or possible pitfalls related to the use of machine learning approaches for mental states estimations applied in pBCI applications, in particular regarding i) linearity of the technique, ii) over/under - fitting issues.

\section{A. Linear or non-linear models: Which to choose?}

The first issue to face with in approaching machine learning techniques to implement $\mathrm{pBCI}$ applications is the choice of the algorithm (model). The first aspect to take into account is related to the linearity of the technique, that is the kind of function (i.e. linear or non-linear) that the specific algorithm uses to maximize the differences between classes. In particular, linear methods are appropriate when limited data and limited knowledge about the data itself are available [18]. However, in the presence of strong noise or outliers, or if the dimensionality of the features space is too high, linear methods can fail. A formal way to increase reliability of the method by manipulating available training data, is to use regularization methods [17]. Regularization allows to limit the influence of outliers or strong noise and the complexity of the discrimination function. However, despite the use of linear or non-linear methods, regularization should always be used. Note that whenever a certain linear classifier does not work well, there could be (at least) two potential causes: (a) either the regularization was not done well (e.g. non-robust estimators are used), or (b) the problem is intrinsically non-linear. If there are large amounts of data, non-linear methods are suitable to find potentially complex structure in the data (i.e. data points not linearly separable). In particular, it has been suggested that when the source of the data to be classified is not well understood, methods that are good at finding non-linear transformations of the features space are recommended. In particular, the suggestion is to use a linear classifier in the appropriate feature space after a proper transformation of the features space. The idea is to apply a linear algorithm in some appropriate non-linear feature spaces calculated by using specific transformation functions (named kernel functions). Despite this idea is simple, it is very powerful. In fact, all beneficial properties (e.g., optimality) of linear classification are maintained, but at the same time the overall classification is non-linear in input space, since feature- and input space are non-linearly related. Best known examples of kernel-based learning machines are Support Vector Machines - SVM techniques [19]. 


\section{B. Over(Under)-fitting}

The two biggest causes of poor performances in using machine learning approaches are overfitting and underfitting. In the former, the model becomes too specific on unique or spurious differences between classes of the training set. The direct consequence is that the model does not perform well over time, and needs repeated calibration sessions [17]. On the contrary, underfitting refers to a technique that can neither model the training data and generalize to new data. Intuitively, underfitting occurs when the model or the algorithm selects too little features to fit the data well enough [20]. One efficient way to detect and possibly avoid such situations is to use the "biasvariance approach" [20]. In particular, the error due to bias is considered as the difference between the expected (or average) prediction of our model and the correct value which we are trying to predict. The error due to variance is taken as the variability of a model prediction for a given data point. Bias is reduced and variance is increased in relation to model complexity. As more and more parameters are added to a model, the complexity of the model rises and variance becomes our primary concern while bias steadily falls and vice versa. There are different techniques that can be used to quantify the model prediction error in order to reduce the under - and overfitting issues, such as i) Adjusted $\mathrm{R}^{2}$, ii) Information Theoretic Techniques, iii) Holdout Sample, iv) CrossValidation and v) Resampling Methods. Between the mentioned methods, in general cross-validation based approaches are preferred. In fact they provide good error estimation with minimal a priori assumptions (e.g. ref. [21]). In addition, linear classifiers are generally more robust and easier to optimize than nonlinear ones, since the latter have limited flexibility (less free parameters to tune) and are, thus, less prone to overfitting. For example, such behavior has been demonstrated for the LDA classifier in the work of Vapnik [22]. Another example has been provided by Aricò and colleagues, that proposed a linear regression algorithm, the automatic stop Stepwise Linear Discriminant Analysis (asSWLDA), a new implementation of the more popular SWLDA, that embed an automatic statistical criterion able to find the optimum number of features to include in the classification model, to mitigate both under and overfitting issues in applications for evaluating mental workload [3], [5]. Huang et al. [23] investigated the factors that can bring to overfitting conditions in Common Spatial Pattern - based applications. In particular, CSP algorithm is widely used to extract discriminative oscillatory features, by removing the signal's strong correlation among the original axes, and the distributions are maximally dissimilar along the new axes. The authors demonstrated both in simulated and in real applications that channel numbers and the correlation between signals influence the generalization of CSP significantly. A larger number of calibrating trials and a longer time length of the trial would prevent overfitting.

\section{PBCI SYSTEM CALIBRATION ISSUE}

Another important issue to mention in the use of pBCI applications in realistic settings is related to the task similarities of the calibration data. In particular, for many pBCI applications it could be difficult to create ecological scenarios to be used to calibrate the classification model. For example, a workload classifier calibrated with classes from a working memory task may not be able to discriminate workload levels in a driving task, where properties of the task and environment will be different. The ascribed issue generated a huge number of works, focusing on the generalization across calibrating and testing tasks, performed in laboratory settings. In this regard, Gerjets et al. [24] proposed a complete review about all the studies that faced with the cross-task classification issue. Anyhow, in real settings classification accuracy could dramatically decrease, depending on several factors not taken into account during the calibration phase (e.g. other mental states involved, noise, etc). The present issue also includes works on generalization across specific electrode montages and days. In this regard, a recent work demonstrated that it could be possible to replace electrodes over dual sessions (thus inducing changes in placement, electromechanical properties, and/or impedance between the electrode and skin surface) without inducing decrement on the accuracy of several machine learning approaches in a binary classification problem [25]. Furthermore, Aricò et al. [3] proposed a system able to evaluate the workload of the operator in a high realistic ATM environment without the need to recalibrate the system up to a month. In addition, loss in system accuracy could depend also by changes in psychophysical state of the user, induced by internal (e.g. individuals who are tired through lack of sleep or recent exercise) and/or external factors (e.g. alcohol, caffeine, [26]). Algorithms that can readapt online classification model could prevent problems due to generalization across time (e.g. [27]). In addition, there are few pBCI related works in which it has been used unsupervised learning i.e. data is unlabeled and the algorithms learn to inherent structure from the input data. The direct advantage of such techniques is to not require calibration data. However, supervised techniques are preferred to the unsupervised ones, since they allow to achieve a higher accuracy [4].

\section{FUTURE TRENDS AND OPEN ISSUES}

Combining results from cognitive neuroscience and systems allowing the detection of specific mental states in real-time, may lead to a new generation of devices (pBCI based) employing to enhance human performance or to fully adapt user interface and consequently improve Human-Machine Interaction to prevent errors risk in operational environments. pBCI systems may be employed to minimize distractions, which include secondary tasks or background noise. For example, a system evaluating a user getting cognitively overloaded, could present less information until the user has free brain cycles to better deal with the details. Another way by which interfaces might adapt themselves is to manage interruptions and triggers based on the user's cognitive state. Real-time mental states estimation will be of high relevance even in Aviation field. For example, the Air Traffic Management interface could be fully automated depending on the actual mental state (e.g. workload, attention, stress) of the 
controller, reducing or even avoiding error commissions [5]. Operating room as well, represents an important application field for pBCI systems, as in [28], demonstrating that the system was able to measure the stress level of a surgeon and alert according to the response type. Mental states measurement could also be employed to assess safety-critical aspects of infrastructure, such as harbors and bridges that require demanding maneuvers [4]. Another area of application is in training procedures, in which displaying learning - related neurophysiological variations to the trainer as well as to the trainee him/herself is expected to improve the training program [8]. This possibility has been explored for pilots [2], air traffic controllers [29], and surgeons [30]. Anyhow, to make such "futuristic" devices usable in operational environments, and more in general in everyday life situations, different steps forward and technological improvements have to be performed.

Firstly, further enhancement in the BCI information transfer rate could be achieved by combining different electrophysiological features together and by moving from binary to multi-class classification [31], [32] in order to improve classification accuracy and enhance the resolution of the measured mental state (e.g. different levels of mental workload or attention). EEG-based neurotechnology for realworld use should profit from further improvement of machine learning and signal processing algorithms, but it will require even a substantial advancement in sensors technology. Advances in wearable, more comfortable and even fashionable sensors technology boosted the field and are expected to contribute further even though there are still major challenges to overcome. To be accepted on the market they need to be well designed, not only in terms of comfort but also aesthetically, and gel-free. In the past few years, electrodes that could be placed so that they are barely visible around the ears have been developed [33]. In addition, different companies are working since few years on the development of increasingly trustworthy dry EEG systems. Currently, there are a number of dry electrode EEG systems available on the market or under further development. These systems do not require the application of gel and sometimes come with a fancy headset. While just few of them showed comparable performance of standard wet EEG systems, dry electrodes need some amount of pressure to maximize conductivity with the skin, which can be uncomfortable or even painful. In addition, such systems seem to be most prone to noise (i.e. electrical interferences, other people and subject's movement artifacts, low-frequency voltage variations caused by the physical movement of the head) with respect to conventional wet systems [34]. On the contrary, for other physiological signals, wearable sensors development and commercialization grew exponentially in the last decade. For example, wristbands that record heart rate or skin conductance are already available offering a high reliability for real life applications [35]. Another important open-issue regarding the application of pBCIs in operational environments is related to the possible confounds induced by "factors unrelated to the variable of interest" (e.g. other involved mental states and/or noise and artifacts). In particular, in the most of the existing studies regarding passive BCIs, experiments are usually conducted in shielded-rooms, where users are shielded from environmental noise (i.e. electrical noise and distractions), and are very limited in talking and moving, as such activities could interfere with the recorded signals, therefore with the evaluation of the considered mental states. However, only the study of $\mathrm{pBCI}$ performed in realistic scenarios - that is within realistic settings and natural behaving-will reveal the actual potential, and also the real challenges of this promising young technology. In particular, in more complex situations (i.e. real settings) there might be other factors unrelated to the mental state of interest, that can cause either the same or even a reversed effect, potentially annihilating the usability of the pBCI-based measure that was previously found viable in a laboratory setting [4]. For example, in a driving scenario with a continuously changing visual background, the alpha rhythm might be completely already "saturated", such that no further decrease due to additional workload can be detected. In other words the expected effect on parietal alpha is not present, such that this feature might be difficult to exploit in an ecological task context. To realize an effective pBCI application in real contexts, it is important to identify those physiological markers or patterns that are specific to the mental states of interest (e.g. independent from the method of elicitation), and at the same time sufficiently general over different contexts (e.g., laboratory versus real world). Another issue in employing pBCI applications in real contexts is related to the increasing number of artifacts recorded in the EEG data. In particular, artifacts can come from i) the subject physiological activity such as eye blinks or muscular behaviors (i.e. correlated with the actual user state) and ii) the environment (i.e. uncorrelated with user state). Artifacts from the first category may be isolated and included as discriminant features to improve the quality of the measure. In this context, the artifact becomes another signal, and can be used as an additional source of information. On the contrary, since the second type of artifacts always decreases the signal to noise ratio, they have to be filtered out from data used for the pBCI application. In this regard, it is critical for artifact removal methods to not introduce unacceptable time delays, to be used online in real-time pBCI applications. A variety of methods have been proposed for EEG artifact removal, such as regression-based (e.g. Gratton algorithm), blind source separation (Independent Component Analysis, ICA), waveletbased. However, many of such methods are not applicable in real-time applications due to their computational complexity, defined as the number of floating point operations (flops) required to execute the algorithm and/or the high number of EEG channels required. In this regard, despite their poorer performance with respect to other methods, regression-based algorithms are preferred for pBCI applications because their lower computational complexity and required EEG channels [36]. Last, but certainly not least issue to highlight, as described previously, is related to similarities between calibration and testing data (see IV.E paragraph), in terms of task (i.e. different calibration and operational tasks) and conditions (e.g. impedance values changing between the electrode and skin surface) over different days. In this regard, research has to take 
several steps forward in developing and/or improving algorithms that i) do not require calibration data (i.e. unsupervised techniques that at the moment suffer of a lower performance with respect to supervised ones [4]) or ii) do not need frequent system recalibrations [3]. Lack of adequate calibration procedures means that $\mathrm{pBCI}$ systems could suffer of low performances as the recording situation or context related data changes from the system calibration ones.

\section{CONCLUSION}

This mini-review highlighted the most important issues and recommendations to consider for the effective implementation and use of pBCI systems in real contexts. Future trends and gaps to bridge in order to make such systems usable in real operational environments have been presented and described. Therefore, we just conclude that, despite the recent innovations in algorithms and technology, pBCI systems are not yet ready to enter the market, unless huge advances in research and technologies to mitigate such limitations. Anyhow, they will represent in the close future a key-tool to augment and burst the Human-Machine Interaction in everyday life, for example enhancing safety standards in operational environments, enhancing adaptive automation systems in assisting the operator in his/her work, or avoiding unsafe mental states, such as mental workload and stress.

\section{REFERENCES}

[1] J. K. Towns, "Human factors studies for injectable combination products: from planning to reporting," PDA J. Pharm. Sci. Technol. $P D A$, vol. 68, no. 4, pp. 347-361, Aug. 2014.

[2] G. Borghini et al., "Measuring neurophysiological signals in aircraft pilots and car drivers for the assessment of mental workload, fatigue and drowsiness," Neurosci. Biobehav. Rev., vol. 44, pp. 58-75, Jul. 2014.

[3] P. Aricò et al., "A passive Brain-Computer Interface (p-BCI) application for the mental workload assessment on professional Air Traffic Controllers (ATCOs) during realistic ATC tasks," Prog. Brain Res. Press, 2016.

[4] B. Blankertz et al., "The Berlin Brain-Computer Interface: Progress Beyond Communication and Control," Front. Neurosci., vol. 10, 2016.

[5] P. Aricò et al., "Adaptive Automation Triggered by EEG-Based Mental Workload Index: A Passive Brain-Computer Interface Application in Realistic Air Traffic Control Environment," Front. Hum. Neurosci., p. 539, 2016.

[6] J. Wolpaw and E. W. Wolpaw, Brain-Computer Interfaces: Principles and Practice. Oxford University Press, 2012.

[7] S. Haufe et al., "Electrophysiology-based detection of emergency braking intention in real-world driving," J. Neural Eng., vol. 11, no. 5, p. 056011 , Oct. 2014

[8] G. Borghini et al., "Quantitative Assessment of the Training Improvement in a Motor-Cognitive Task by Using EEG, ECG and EOG Signals," Brain Topogr., Jan. 2015.

[9] K. Mandrick et al., "Neural and psychophysiological correlates of human performance under stress and high mental workload," Biol. Psychol., vol. 121, no. Pt A, pp. 62-73, Dec. 2016.

[10] G. Vecchiato et al., "Investigation of the effect of EEG-BCI on the simultaneous execution of flight simulation and attentional tasks," Med. Biol. Eng. Comput., vol. 54, no. 10, pp. 1503-1513, Oct. 2016.

[11] M. K. Scheffers and M. G. Coles, "Performance monitoring in a confusing world: error-related brain activity, judgments of response accuracy, and types of errors," J. Exp. Psychol. Hum. Percept. Perform., vol. 26, no. 1, pp. 141-151, Feb. 2000.

[12] E. Niedermeyer and F. H. L. da Silva, Electroencephalography: Basic Principles, Clinical Applications, and Related Fields. Lippincott Williams \& Wilkins, 2005.
[13] J. Toppi et al., "Investigating Cooperative Behavior in Ecological Settings: An EEG Hyperscanning Study," PLOS ONE, vol. 11, no. 4, p. e0154236, Apr. 2016.

[14] Y. Sun et al., "Discriminative analysis of brain functional connectivity patterns for mental fatigue classification," Ann. Biomed. Eng., vol. 42, no. 10, pp. 2084-2094, Oct. 2014.

[15] S. I. Dimitriadis et al., "Cognitive workload assessment based on the tensorial treatment of EEG estimates of cross-frequency phase interactions," Ann. Biomed. Eng., vol. 43, no. 4, pp. 977-989, Apr. 2015.

[16] A. Gundel and G. F. Wilson, "Topographical changes in the ongoing EEG related to the difficulty of mental tasks," Brain Topogr., vol. 5, no. 1, pp. 17-25, 1992.

[17] S. Lemm et al., "Introduction to machine learning for brain imaging," NeuroImage, vol. 56, no. 2, pp. 387-399, May 2011.

[18] J. R. Wolpaw et al., "Brain-computer interface technology: a review of the first international meeting," IEEE Trans. Rehabil. Eng., vol. 8, no. 2, pp. 164-173, Jun. 2000.

[19] G. Mountrakis et al., "Support vector machines in remote sensing: A review,” ISPRS J. Photogramm. Remote Sens., vol. 66, no. 3, pp. 247259, May 2011.

[20] S. Fortmann-Roe, Understanding the bias-variance tradeoff. 2012.

[21] D. Bzdok and B. T. T. Yeo, "The Future of Data Analysis in the Neurosciences," ArXiv160803465 Q-Bio Stat, Aug. 2016.

[22] V. N. Vapnik, The Nature of Statistical Learning Theory. New York, NY: Springer New York, 2000.

[23] G. Huang et al., "Model based generalization analysis of common spatial pattern in brain computer interfaces," Cogn. Neurodyn., vol. 4, no. 3, pp. 217-223, Sep. 2010.

[24] P. Gerjets et al., "Cognitive state monitoring and the design of adaptive instruction in digital environments: lessons learned from cognitive workload assessment using a passive brain-computer interface approach," Front. Neurosci., vol. 8, Dec. 2014.

[25] J. R. Estepp and J. C. Christensen, "Electrode replacement does not affect classification accuracy in dual-session use of a passive braincomputer interface for assessing cognitive workload," Front. Neurosci., vol. 9 , p. $54,2015$.

[26] P. Aricò et al., "Influence of P300 latency jitter on event related potential-based brain-computer interface performance," J. Neural Eng., vol. 11, no. 3, p. 035008, Jun. 2014.

[27] F. Schettini et al., "Self-calibration algorithm in an asynchronous P300based brain-computer interface," J. Neural Eng., vol. 11, no. 3, p. 035004, Jun. 2014

[28] D. G. Duru et al., "Assessment of surgeon's stress level and alertness using EEG during laparoscopic simple nephrectomy," in 2013 6th International IEEE/EMBS Conference on Neural Engineering (NER), 2013, pp. 452-455.

[29] P. Aricò et al., "Air-Traffic-Controllers (ATCO) - Neurophysiological analysis of training and workload ATCO," Ital. J. Aerosp. Med., vol. 12, pp. 18-35, Apr. 2015.

[30] G. Borghini et al., "Neurophysiological measures for users' training objective assessment during simulated robot-assisted laparoscopic surgery," in 2016 38th Annual International Conference of the IEEE Engineering in Medicine and Biology Society (EMBC), 2016, pp. 981984.

[31] R. Venkatesan and M. J. Er, "A novel progressive learning technique for multi-class classification," Neurocomputing, vol. 207, pp. 310-321, Sep. 2016.

[32] P. Arico et al., "Towards a multimodal bioelectrical framework for the online mental workload evaluation," in 2014 36th Annual International Conference of the IEEE Engineering in Medicine and Biology Society (EMBC), 2014, pp. 3001-3004.

[33] D. Looney et al., "Ear-EEG: User-Centered and Wearable BCI," pp. 41-50, 2014.

[34] J. Saab et al., "Simultaneous EEG Recordings with Dry and Wet elecTrodes in Motor-Imagery," presented at the Verlag der Technischen Universität Graz, Graz, Austria, 2011, pp. 312-315.

[35] A.-M. Brouwer et al., "Evidence for effects of task difficulty but not learning on neurophysiological variables associated with effort," Int. J. Psychophysiol., vol. 93, no. 2, pp. 242-252, Aug. 2014.

[36] J. A. Urigüen and B. Garcia-Zapirain, "EEG artifact removal - state-ofthe-art and guidelines," J. Neural Eng., vol. 12, no. 3, p. 031001, 2015. 